\title{
REDUCCIÓN DE LA CONTAMINACIÓN VISUAL COMO ESTRATEGIA DE \\ RESILIENCIA URBANA
}

\section{REDUCTION OF VISUAL CONTAMINATION AS AN URBAN RESILIENCE STRATEGY}

Sonia Emilia Leyva Ricardo, MsC. Máster en Contaminación Ambiental, mención en Gestión Ambiental y Protección de los Recursos Naturales (Cuba). Docente de la Universidad Tecnológica Equinoccial, sede Santo Domingo, Ecuador. sonia.leyva@ute.edu.ec

José Armando Pancorbo Sandoval, Ph.D. Doctor en Administración y Dirección de Empresas (Cuba). Docente de la Universidad Tecnológica Equinoccial, sede Santo Domingo, Ecuador. jose.pancorbo@ute.edu.ec Efrain Eduardo Vizuete Jaramillo, MsC. Máster en Ciencias de Recursos Naturales (México). Docente del Instituto Tecnológico de Sonora, México. efra.vzt@gmail.com

\section{ARTÍCULO DE INVESTIGACIÓN}

Recibido: 11 de junio de 2018.

Aceptado: 19 de julio de 2018.

\section{RESUMEN}

El objetivo central de la presente investigación es definir una propuesta de índices que permitan evaluar la contaminación visual en la zona comercial de Santo Domingo, en función de variables arquitectónicas, estructurales, de publicidad e imagen y ambientales especialmente como es el uso de los espacios verdes, para lo cual se parte de la visión desde la resiliencia urbana. Para el cumplimento de este proyecto de investigación, se realiza una revisión de las actuales tendencias en la gestión de la resiliencia urbana y específicamente de cómo evaluar el nivel de contaminación 
ambiental en ciudades ecuatorianas, aplicándose herramientas y técnicas que posibilitan el análisis integrado de todas las variables de estudio para realizar una propuesta que permita evaluar el grado de contaminación visual en la zona de estudio seleccionada para el estudio.

Palabras clave: resiliencia urbana, contaminación visual, gestión ambiental, sistema de indicadores

\section{ABSTRACT}

The main objective of the present investigation is to define a proposal of indexes that allow to evaluate the visual contamination in the commercial zone of Santo Domingo, based on architectural, structural, advertising and image and environmental variables, especially as it is the use of green spaces, which is part of the vision from the urban resilience. For the fulfillment of this research project, a review is made of current trends in urban resilience management and specifically how to assess the level of environmental pollution in Ecuadorian cities, applying tools and techniques that enable the integrated analysis of all the study variables to make a proposal that allows to evaluate the degree of visual contamination in the study area selected for the study.

Keywords: urban resilience, visual pollution, environmental management, indicators system

\section{INTRODUCCIÓN}

En la actualidad, más del $50 \%$ de la población mundial reside en zonas urbanas, y debe seguir creciendo hasta el 66\% para 2050. La rápida urbanización en América Latina, en un escenario caracterizado por las complejas características de sus ciudades contribuye al aumento al mismo tiempo de las vulnerabilidades y el riesgo (UNISDR, 2018).

Las características físicas y espaciales de las zonas urbanas, la vulnerabilidad socioeconómica de sus habitantes, las deficiencias de las capacidades institucionales y los desafíos ambientales son algunos de los factores impulsores del riesgo que se desarrollan en la compleja situación en la que se encuentran las ciudades.

La conformación física y natural del espacio urbano posee un equilibrio y armonía que permiten identificar a simple vista la presencia de factores que intervienen y afectan, como puede ser el exceso de publicidades, erróneas carta de colores de las construcciones, inadecuadas áreas verdes, inadecuadas normas urbanas, entre otros. 
Este tipo de intervención es más evidente en zonas de la ciudad con marcada actividad comercial y alta concentración de población, como es el caso de la ciudad de Santo Domingo de los Colorados, Ecuador, la cual se considera una de las ciudades con mayor densidad comercial con una compleja y arcaica estructura logística que afecta el valor hedónico del espacio público y privado (Arias, 2001).

Dentro de las definiciones de contaminación visual relacionada con el espacio público urbano, encontramos a autores como Etulain, et al. (1996), UNNE (2006) y Trujillo (2010), los cuales coinciden en que se refiere a una afectación que distorsiona el paisaje urbano y en la que intervienen otros factores además de los publicitarios.

El elemento atípico es un concepto que emerge de evidenciar visualmente en el nivel peatonal, objetos en el espacio urbano que no hacen parte de su conformación física original y que desde el punto de vista estético y por sus mismas características de número, ubicación, deterioro o falta de mantenimiento, entre otras, generan una presencia que afecta negativamente el espacio público; esta afectación se puede apreciar en una fachada saturada de avisos comerciales no autorizados, en superficies deterioradas y sucias, en cableados instalados sin ningún tipo de consideración estética o técnica o en la instalación de antenas de telecomunicaciones, entre otras afectaciones.

\section{REVISIÓN TEÓRICA}

\section{Contaminación visual y resiliencia urbana.}

La contaminación visual puede ser comprendida desde la visión de la resiliencia urbana, ya que, según este concepto, la cual la resiliencia de una ciudad va a depender directamente de "la capacidad de todos los componentes físicos del sistema, incluidos los edificios y las infraestructuras de transporte, para absorber los daños producidos por una perturbación externa y recuperar rápidamente el nivel previo a la perturbación" (Matthews, Osorio, Cavallaro, 2018). Por tanto, la contaminación visual es un factor degradante del sistema social y técnico de la urbe $y$, por ende, se requiere de comprender la necesidad de la resiliencia urbana como vía para mantener y fortalecer la funcionalidad del sistema urbano.

Una vez analizado diferentes conceptos o puntos de vista los autores del presente proyecto de investigación proponen la siguiente definición... La contaminación visual se 
origina en el desequilibrio del paisaje por el incumplimiento de las normas urbanas y por la ausencia de una política técnica y cultural adecuada en el escenario en estudio.

Por tanto, se hace necesario la necesidad de un enfoque integrado, multinivel y con la participación de múltiples interesados para crear resiliencia ante una serie de perturbaciones y tensiones a diferentes niveles y escalas en sistemas complejos como es la actividad comercial minorista tanto la formal como la informal.

La calidad del ambiente urbano depende en gran medida del equilibrio de las fuerzas que interactúan en el espacio público, como es el caso de los muebles urbanos, cartas de colores, diseño de publicidad y de las infraestructuras urbanas. Las ciudades ecuatorianas no están ajenas a esta situación, pero en algunos casos como es el de Santo Domingo de los Tsáchilas, se alcanzan niveles muy altos de contaminación visual.

Uno de los problemas que enfrenta Santo Domingo es el uso extensivo de publicidad en los espacios urbanos, los cuales invaden el poco espacio que hay en la ciudad y en muchos de los casos por mal uso o por olvido han contribuido con la gran problemática en la ciudad como es la existencia de espacios abandonados, que se denominan en urbanismo como espacios basuras. Por otra parte, la ciudad ha crecido de forma desordenada, convirtiéndose en lo que se denomina ciudad difusa, que no es más que las urbes que no tiene equilibrados sus diferentes áreas sociales y productivas.

En la actualidad, es una de las provincias de mayor proyección económica y demográfica del país, especialmente en el casco urbano, por ser una ciudad que en un lapso de tiempo muy corto ha crecido de forma impresionante y desordenada que ha desencadenado un crecimiento desigual afectando principalmente el paisaje natural y urbano (Plan de desarrollo de Santo Domingo, 2015-2030).Uno de los proyectos más ambiciosos de la provincia está en la creación de un puerto terrestre el cual tendrá la capacidad para prestar la totalidad de los servicios públicos y privados relacionados con el transporte, almacenamiento de carga y control aduanero de los productos que ingresan y salen del país. Esto tendrá un impacto positivo en la economía local y regional y por ende por estar inmerso en el comercio obligadamente irá de la mano con la publicidad que esta pueda promocionar cada empresa (Plan de desarrollo de Santo Domingo, 2015-2030).

Santo Domingo se ubica como un nodo de interconexión nacional, pues su posición estratégica en el territorio nacional viene dada por la articulación Sierra - Costa que le ha permitido la convergencia de cuatro ejes viales arteriales y un eje colector nacional. 
Esta es una de las características que ha dado origen a importantes flujos de migración y crecimiento poblacional.

Un plan director urbano puede contribuir a la resiliencia, es decir, ser capaz de resistir y mitigar el impacto de los eventos y, ser flexible a los cambios; aprovechando el trabajo en red y la capacidad de reorganizar las estructuras y recursos disponibles, siempre y cuando considere los factores que afectan al ecosistema urbano (Rivera y Gabriel, 2013).

La contaminación ambiental, lamentablemente, no es considerada por muchos autores como una variable de resiliencia; a pesar de que sí afecta la calidad del ciudadano. Las principales dificultades en la integración de la resiliencia en una evaluación de impacto ambiental urbana están asociadas con la incertidumbre de las amenazas y de la dificultad de preverlas; así como de que sus consecuencias no son medibles cuantitativamente al ser de efecto a mediano y largo plazo en la salud mental de los ciudadanos.

Por otra parte, los instrumentos de evaluación de sostenibilidad se diseñan con condiciones de partida conocidas, mientras que la contaminación visual no es predecible en sus efectos a corto plazo. En este sentido es importante propiciar el diseño sostenible para reducir las vulnerabilidades e inequidades de los espacios urbanos, como pueden ser fachadas y colores inapropiados, deben dirigirse a equilibrar las brechas existentes a nivel ambiental, social y económico mediante la aplicación de sistemas de indicadores como el que se propone en este proyecto para reducir los daños asociados por inapropiados diseños urbanos (Diestra, 2017).

\section{MATERIALES Y MÉTODOS}

Propuesta para la evaluación de la contaminación visual en la av. Quito y 29 de mayo en la zona comercial de Santo Domingo.

Para el desarrollo del proyecto de investigación se aplicaron tres herramientas que estructuraron a su vez un procedimiento del modo siguiente: 
Figura 2. Etapas del procedimiento

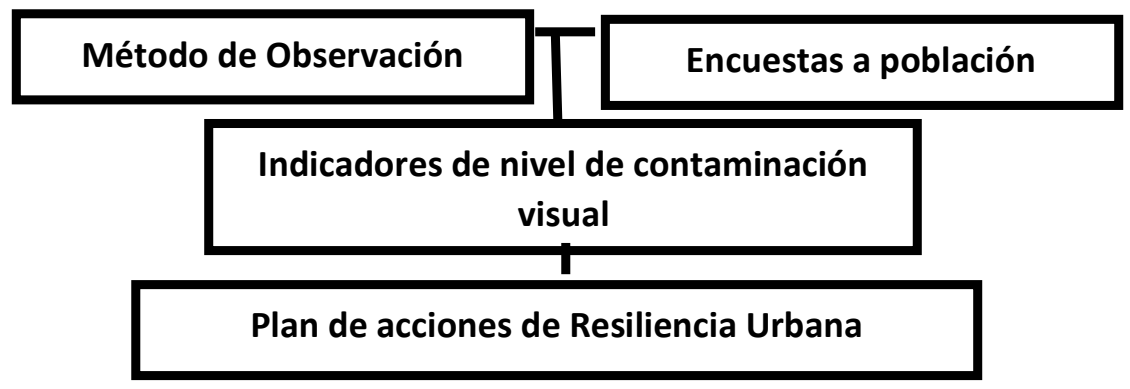

Fuente: Elaboración propia

\section{Técnica de observación}

Se parte de identificar la presencia de elementos atípicos en el espacio urbano para lo cual se confecciona un mapa de la zona en estudio, se realiza un inventario a través de una identificación visual derivada del recorrido peatonal y de los registros de base de datos del GAD municipal, registros fotográficos y geográfico, de tal modo, esta etapa propicia la información base que permite la posterior aplicación del sistema de indicadores de nivel de contaminación visual. En la siguiente figura se expone las etapas de aplicación de la técnica de observación.

Figura 3. Etapas de aplicación de la Técnica de Observación.

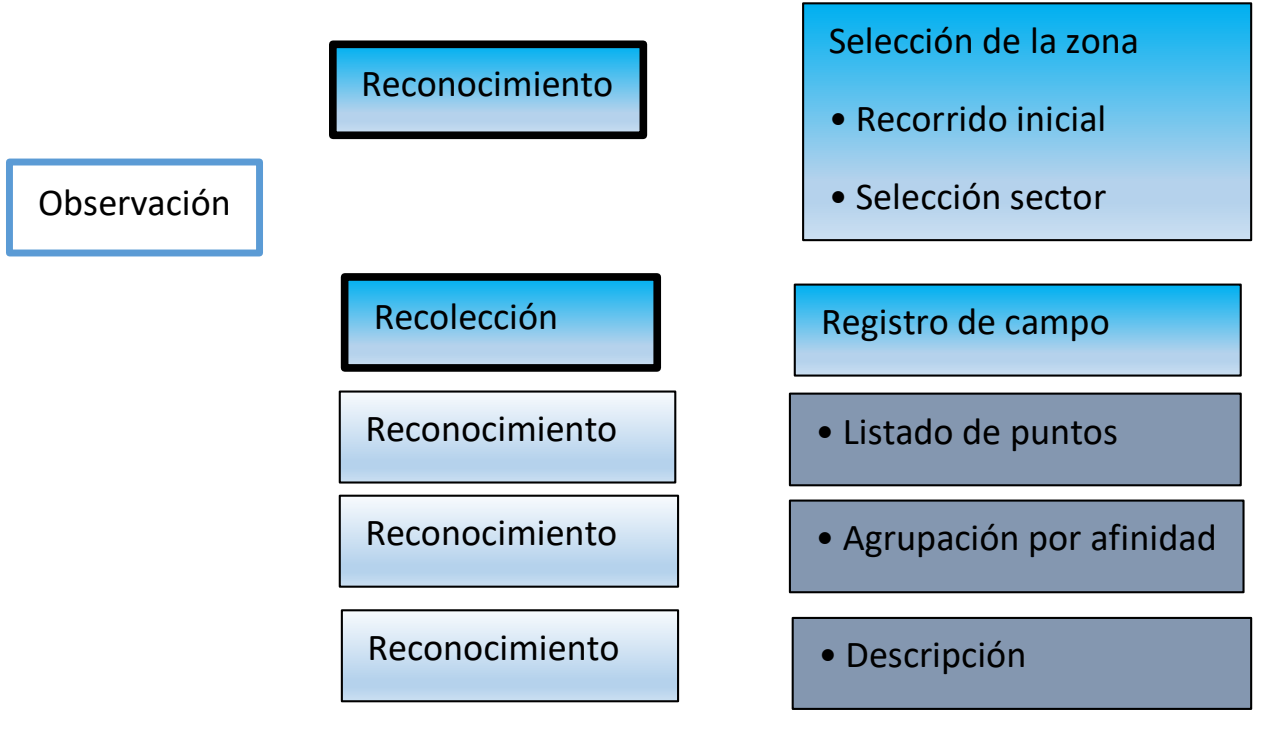

Fuente: Elaboración propia 


\section{Entrevistas}

Se aplicaron un total de 134 encuestas partir de la población existente en el centro histórico de la ciudad, considerados la modalidad de encuestas por conveniencia, las cuales se aplicaron en la zona de estudio, específicamente en la Av. Quito y 29 de mayo para tener una percepción general de los ciudadanos y dueños de locales comerciales en relación con la contaminación visual.

Por razones de espacio, se exponen a continuación las principales conclusiones derivadas de la aplicación de las encuestas. El 90\% los entrevistados han manifestado que perciben que el exceso de publicidad genera en ellos malestar, incomodidad y distracción lo cual si cambia el estado de ánimo en estas personas y puede repercutir en un cansancio físico y mental al tener que esforzarse por estar más concentrados en sus labores diarias.

Mediante la entrevista que se realizó a los transeúntes se determinó que el 22\% piensa que la alcaldía es la encargada de controlar la contaminación visual, mientras que el $78 \%$ cree que el Ministerio del Ambiente es el encargado de esa función. En este caso puede existir desinformación acerca de quién es el rector en el tema del control de la contaminación visual, y es por eso que la población puede contestar esta pregunta por intuición y no por verdadero conocimiento. Si bien se conoce acerca de las competencias que se le da a cada institución, en la actualidad la alcaldía solo está limitada al derecho de verificar y constatar los permisos de colocación de publicidad en locales comerciales y sancionar como corresponde la ley por no tener este tipo de permisos. Actualmente solo el Ministerio del Ambiente puede controlar este tipo de contaminación ya que es el órgano rector con todas sus facultades llamado a velar por esta forma de contaminación. El municipio podría controlar este tipo de contaminación, sólo si el MAE les da el aval mediante una certificación que permita controlar y sancionar este tema en estudio.

De igual forma, la población está consciente que en la Av. Quito y 29 de mayo está afectando el exceso de publicidad, es decir, tienen la percepción de que cuando recorren el lugar la publicidad abusa de los espacios, en tamaños y colores. A pesar de que existen leyes que pueden controlar el uso adecuado de publicidad y el ornato en la ciudad, ha faltado mayor aplicación de la ley en las mismas, también mediante estas encuestas se pudo constatar que la población no tiene conocimientos de estas leyes, o sea, por parte de las autoridades falta mayor socialización de las leyes u ordenanzas 
municipales, ya que si los ciudadanos tienen noción de esta situación sería más fácil que ellos puedan tomar acciones correctivas cuando sucedan este tipo de problemas.

Un resultado interesante es la valoración sobre los efectos de la contaminación visual en su salud, siendo los más importantes con el 18\% el estrés por saturación de elementos y colores, seguido con el $22 \%$ los accidentes ocasionados por obstrucción visual al conductor y la más representativa con el $35 \%$ la afectación de la estética del paisaje urbano.

Este tipo de factores que pueden ser afectados por la contaminación visual hoy en día son más que una realidad, muchos de los accidentes automovilísticos son producto de elementos que distraen a los conductores, pero en este caso es muy importante a considerar que la población está consciente de que la contaminación visual en Santo Domingo afecta significativamente la estética del paisaje urbano, es decir, los habitantes saben que el entorno que los rodea está en mal estado por diversos factores, como la publicidad, los colores y la falta de infraestructura urbana que permite darle una mejor cara a la ciudad.

Un interesante resultado es que el $37 \%$ piensa que la contaminación visual es sancionada, mientras que el $63 \%$ de la población encuestada piensa que no es sancionada por la ley. Esto quiere decir que hay un desconocimiento generalizado acerca de las leyes u ordenanzas municipales que pueden regir sobre el asunto de la contaminación visual, es decir, hay poca información disponible o la divulgación de estas normativas es muy poca y es por eso el desconocimiento de la población.

Los autores de la presente investigación al respecto consideran que es evidente la importancia de desarrollar un plan de resiliencia urbana que posibilite una evaluación de referencia cuyo fin radica, precisamente, en medir la capacidad de recuperación después de los efectos negativos del daño al paisaje urbano de los problemas asociados, por ejemplo, a la excesiva publicidad o edificios. De igual modo se requiere de un programa que desde el plan director de la ciudad considere las afectaciones a la movilidad peatonal y de carros, por exceso de publicidad, antenas y edificios en mal estado o el denominado espacio basura.

También, es importante resaltar que la mayoría de la población piensa que la principal consecuencia de la contaminación visual es la afectación de la estética paisajística, lo cual quiere decir, que las personas están conscientes que el exceso de publicidad le 
quita la buena imagen a Santo Domingo, convirtiéndola en una ciudad por la cual muchos visitantes se llevan una mala percepción y puede ser transmitida a otros.

La uniformidad de colores en edificios y viviendas es un factor importante para la imagen y la estética de una ciudad, en la actualidad muchas personas en un porcentaje casi equitativo están de acuerdo y otra, por lo contrario, con la uniformidad de los colores en las fachadas e viviendas y edificios; esto puede ser por motivos de cultura, ya que son personas que crecieron con el concepto de que una casa puede ser pintada con todos los colores que a ellos les parezca conveniente sin tomar en cuenta el factor paisajístico de una ciudad, mientras que otros ya han tomado conciencia y han tratado de homogenizar los colores para tener una mayor uniformidad en sus barrios.

Es por eso la importancia de conocer la perspectiva de las personas ante la contaminación visual, ya que permite saber cómo se siente la población, qué nivel de conocimientos y qué reacciones puede tener ante este problema, y de esta forma se puede considerar no solo la parte técnica, sino también la parte social como principal factor para cambiar la mala imagen de la ciudad, también tomando en consideración el mismo beneficio comercial y turístico de la ciudad, ya que la imagen es la principal carta de presentación ante las demás ciudades y provincias de las cuales Santo Domingo ha dependido durante muchos años. Con esto se ha conseguido entender que en la actualidad, la población de Santo Domingo, ya conoce esta problemática y que no se siente cómoda al ver tanta publicidad, cables eléctricos y la amplia gama de colores lo cual repercute de forma directa $o$ indirecta en los pobladores con incomodidad 0 malestar, influyendo en el cambio de la forma de vivir cotidiana.

Sistema de indicadores de contaminación visual

El método propuesto se basa en el análisis de tres elementos urbanos: la manzana, la avenida y los espacios abiertos, ya que se consideran según estudios precedentes, el sustento para la ubicación de las edificaciones o elementos arquitectónicos, al ser las variables que mayormente generan las imágenes, de las cuales se alimenta la percepción visual. (Morella, et al. 2003).

Por lo tanto, los indicadores se estructuran del modo siguiente: la forma (reticular, lineal, orgánica, radial), la relación de espacios llenos y vacíos (porcentajes de llenos y vacíos), figura-fondo de manzana y de grano (grueso, fino, mezcla, borroso), la textura (en relación con las alturas de las edificaciones: regular, media, irregular, muy irregular), los 
hitos arquitectónicos y no arquitectónicos (religioso, educativo, cultural y otros) y el tejido (denso, medio, disperso u otro).

Se considera que el estudio de la avenida como espacio tridimensional de conexión, se debe realizar a través del levantamiento de imágenes en donde se validen variables como: estado del pavimento, trazado de aceras, densidad de cableado eléctrico, volumen de la publicidad, uso de áreas verdes, estado y diseño del mobiliario urbano, formato del comercio informal, continuidad de fachadas, materiales y acabados y mantenimiento, medidas a partir de los indicadores: la forma (recta, quebrada, continua, curva), la función predominante (vehicular, peatonal), la estructura parcelaria (grande, mediana, ausencia), volumetría predominante (entrante, saliente, plana), color predominante (cálidos, fríos, mixtos u otros), tipología predominante (tradicional, moderna, interés social), umbral de cerramiento (total, parcial, bajo, vacío), perfil de la calle (regular e irregular), pórticos en edificaciones (continuo, discontinuo, curvo y otros).

Para evaluar la calidad ambiental de la imagen se diseñaron índices, las cuales fueron propuestas por Morella Briceño A. y Beatriz Gil S., a las cuales se les hizo ciertas adaptaciones para poder incluir la parte ambiental en relación con cada uno de los elementos urbanos antes mencionados. A su vez, estos índices cuentan con una ficha técnica de evaluación de la contaminación visual, como respaldo que considera cada elemento urbano en función de sus variables, el cual en conjunto con un manual de evaluación permitirán darles una valoración real a los índices propuestos.

Se ha establecido una valoración manejando un puntaje $(1,2,3)$ desde el nivel más bajo de calidad ambiental (Deficiente) observado en estos elementos urbanos hasta el nivel más alto (Excelente). Estos tres niveles sintetizan las cualidades perceptuales y de imagen (igualdad, proximidad, regularidad, simplicidad, simetría, identidad y estructura) que existen en un lugar. En los niveles bajos se observa la ausencia de las cualidades perceptuales y de imagen urbana, es decir, la contaminación visual se considera alta, y por el contrario, cuando estas cualidades están presentes en los elementos urbanos se habla de niveles bajos de contaminación visual.

Esta metodología permite cuantificar con cierta precisión acompañada de un registro fotográfico el comportamiento cualitativo de las variables de los elementos de la imagen urbana. Es importante señalar que la valoración se orienta sobre parámetros subjetivos, sin embargo, la intención es que éstos puedan ser traducidos a valores cuantitativos a ser considerados dentro de normativas y ordenanzas urbanas. 
Una vez evaluados cada uno de los elementos urbanos, se diseñó una tabla síntesis, la cual representa el nivel de calidad ambiental de la imagen urbana, representada tanto en los diferentes sectores de análisis, como en su valor total de la calidad ambiental de la imagen urbana.

Ficha técnica de evaluación de la contaminación visual

La ficha técnica de evaluación de la contaminación visual permite registrar y evaluar cada elemento urbano en función de sus variables, lo cual contribuirá a simplificar la evaluación en los índices propuestos, teniendo previamente un criterio de evaluación apoyado en la ficha técnica de evaluación.

Escala de índice de la contaminación visual

Como propuesta para la evaluación de la contaminación visual se construye el siguiente cuadro con sus rangos:

Tabla 2. Escala de contaminación visual.

\begin{tabular}{|l|l|l|}
\hline \multirow{2}{*}{ Rango } & \multicolumn{2}{|l|}{ Criterios Generales } \\
\cline { 2 - 3 } & Escala & Descripción \\
\hline $0-7$ & & Nivel de contaminación visual alta \\
\hline $8-14$ & Nivel de contaminación visual media \\
\hline $15-21$ & & Nivel de contaminación visual baja \\
\hline
\end{tabular}

Fuente: Elaborado por el autor Efrain Vizuete

Fórmula de Índice de Evaluación de la Contaminación Visual

Para obtener un resultado numérico de la contaminación visual que permita comparar en la escala anteriormente descrita, se ha propuesto la siguiente fórmula matemática:

Donde:

ÍEcv = Índice de Evaluación Contaminación Visual

nv $=$ Sumatoria del total de los niveles de valoración

= Mínima expresión del coeficiente de evaluación (10) 
Aplicación del método para evaluar la Calidad Ambiental Urbana a partir de la Imagen Urbana en la Av. Quito y 29 de mayo de Santo Domingo.

El levantamiento de la información de campo está basado en la recolección de datos observados en el sitio. La elección del sector de análisis obedece a que las características generales del lugar incluyen áreas de comercio, áreas de nuevo desarrollo económico y desarrollo espontáneos categorizados en las normativas urbanas como ventas ambulantes, además de poseer continuidad espacial entre ellos. A saber: Áreas comerciales: Sector de la Av. Quito (AQ); y Av. 29 de mayo (AM).

Figura 3. Mapa de ubicación de la zona de estudio.

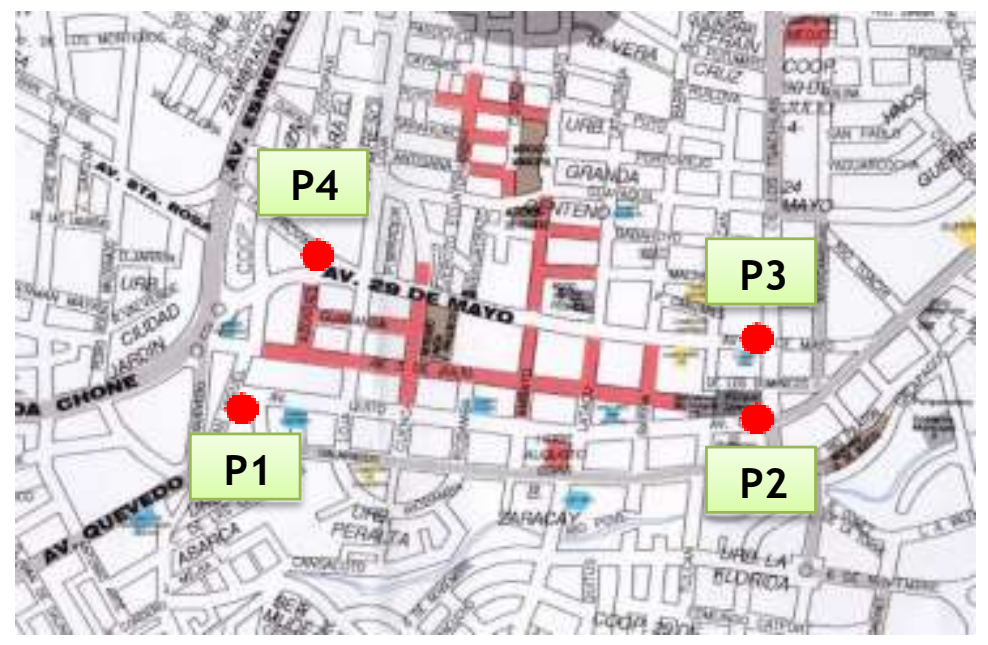

Fuente: Elaboración propia.

Levantamiento de Fichas

En la implementación del método en el análisis del sitio será necesario la elaboración de índices (descritos anteriormente) para cada uno de los elementos urbanos: la manzana, la avenida y los espacios abiertos.

Una vez recolectada la información de los índices, los datos son puestos en funciones de la fórmula (descrita en el método) para determinar la valoración de la calidad ambiental.

Valoración de la Calidad Ambiental de la Imagen Urbana

Una vez obtenido los resultados mediante la fórmula de Índice de Evaluación de la Contaminación Visual propuesta por los autores se adjuntarán los resultados en una matriz general que determinará de forma macro la calidad ambiental de la imagen 
urbana en los sectores de estudio, según los códigos que se hayan asignado a cada lugar de estudio.

Tabla 3. Universidad Tecnológica Equinoccial. Contaminación visual urbana.

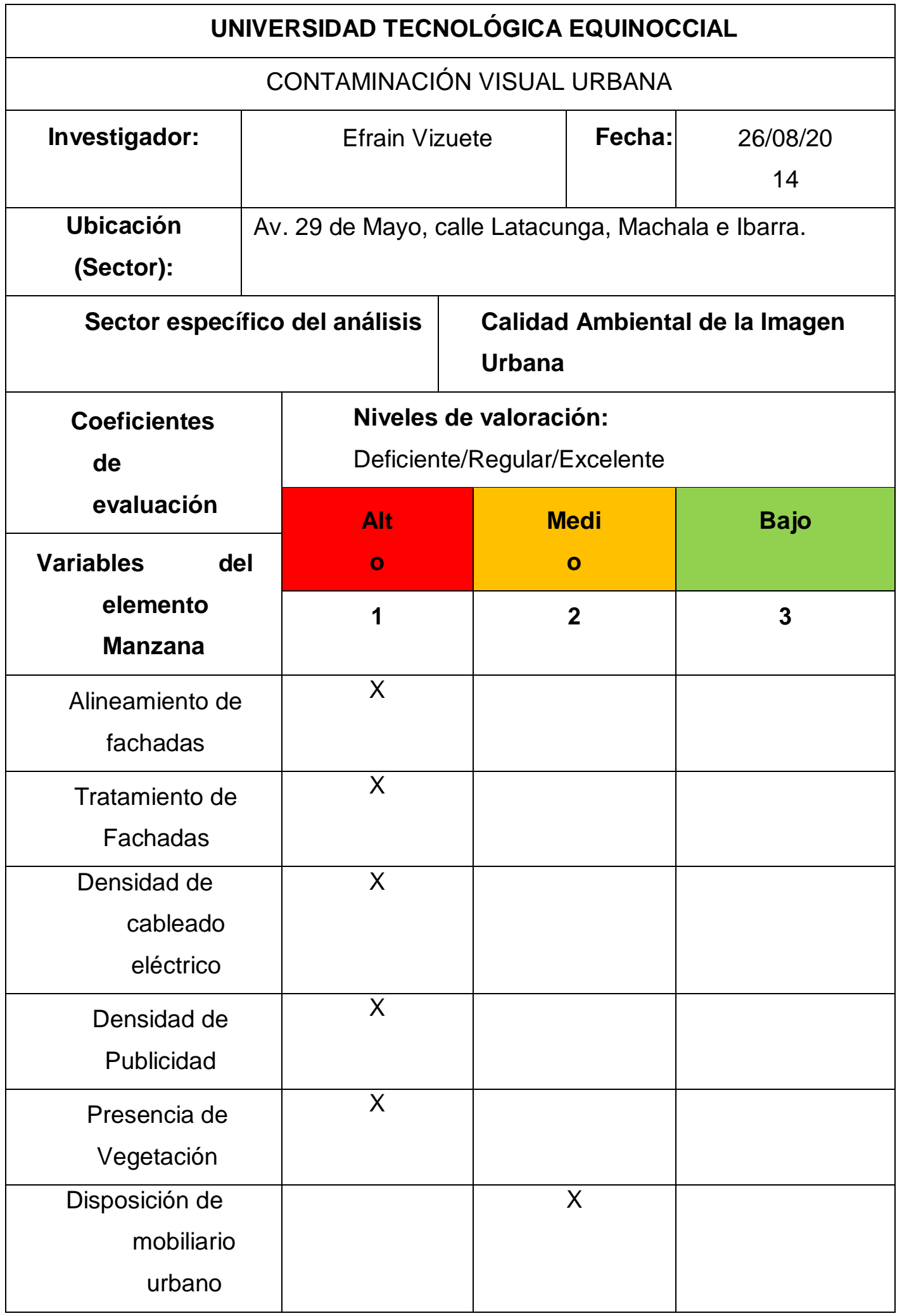




\begin{tabular}{|c|c|c|c|}
\hline $\begin{array}{c}\text { Ventas Ambulantes } \\
\text { Estado de bordes y } \\
\text { aceras }\end{array}$ & $\mathrm{X}$ & $\mathrm{X}$ & \\
\hline $\begin{array}{c}\text { Materiales y } \\
\text { acabados }\end{array}$ & & $\mathrm{X}$ & \\
\hline Mantenimiento & & $\mathrm{X}$ & \\
\hline Total & 6 & 8 & \\
\hline
\end{tabular}

Fuente: Elaborado por el autor Efrain Vizuete

\section{ANÁLISIS DE LOS RESULTADOS}

La medición de la contaminación visual en este sector es alta, esto es debido a que la mayoría de sus problemas se encuentran en sus fachadas, la publicidad, el tendido eléctrico y en sus bordes y aceras. Para esta manzana se obtuvo un valor de 4 que está comprendido en el rango de 0 a 7, lo que significa que el nivel de contaminación en esta manzana es alto, esto se da debido a que la mayoría de los elementos o casi en su totalidad se encuentra en deficiente estado.

Como se puede observar en el inventario que se realiza, gráfico del área en estudio, la mezcla de usos ya sea con fines comerciales o de vivienda, es muy alto y con serios problemas de mantenimiento constructivo y con daños ocasionados por la humedad, debido al clima extremo de la región. El alineamiento de fachadas es discontinuo porque existen entrantes y salientes de edificaciones; sin embargo, la tipología edificada es básicamente tradicional en el sector.

Las fachadas que presenta esta manzana es irregular, es decir, la presencia de edificios altos y bajos es muy notorio lo cual hace perder la uniformidad de alturas en cuanto a las edificaciones, del mismo modo estas fachadas no tienen un buen tratamiento, muchas aún se encuentran en obra negra, sin enlucir o sus acabados aún son deficientes.

\section{El Plan de Resiliencia Urbana}

A partir de estos resultados se propone incluir en el perfil de resiliencia, el diagnóstico y muestra, el estado de la ciudad en lo referente a la variable contaminación visual, ya que se considera a juicio de los autores del presente proyecto de que la información obtenida constituye la base de trabajo a partir de la cual los actores locales podrán tomar decisiones adecuadas a su perfil y sus capacidades para lograr la mejora de la 
resiliencia en lo referente a la reducción de la contaminación visual. Con ese último objetivo se definirán los objetivos estratégicos y las líneas de acción inmediata, ya que en este caso los riesgos a acotar son los referidos al socio organizativos, básicamente a los vandalismos, normas urbanas inadecuadas, ausencia de cultura urbana y sobre todo a problemas con la personalidad urbana.

En el estudio se identifica una tipología de contaminación visual pocas veces tratada en la literatura de riesgos ambientales que es la lumínica, ya que no solo afecta a los seres humanos, sino que es nueva amenaza para la polinización; en este caso, la Universidad de Berna (Suiza) los investigadores han detectado que la contaminación lumínica afecta a la polinización nocturna lo que conlleva a una reducción en los frutos, por ejemplo, que puedan dar una planta; el cual también detectó que esta disminución no puede ser equilibrada por los polinizadores diurnos. El hecho de que las noches ya no sean tan oscuras modifica la naturaleza y, por ende, en el plan de resiliencia debería incluirse como un potencial riesgo en el equilibrio del ecosistema de la región en estudio.

De la aplicación del procedimiento y de las encuestas se identifica una importante variable que se denomina elemento atípico, lo cual es un concepto que identifica a los objetos en el espacio urbano que no pertenecen a la estructura espacial urbana original y que, desde el punto de vista estético, y por sus mismas características de número, ubicación, deterioro o falta de mantenimiento, entre otras generan una presencia que afecta negativamente el espacio público; esta afectación se puede apreciar en una fachada saturada de avisos comerciales no autorizados, en superficies deterioradas y sucias, en cableados instalados sin ningún tipo de consideración estética o técnica o en la instalación de antenas de telecomunicaciones sobre la cubiertas de los edificios. El término elemento atípico, asociado con la contaminación visual, proviene de la ponencia presentada por Rivera (2013) al IV Congreso Colombiano sobre Calidad del Aire.

El elemento atípico, por lo tanto, es el factor que en un plan de resiliencia urbana debe considerarse ya que es el origen de posibles daños visuales, porque se pueden presentar varios sitios con el mismo tipo de afectación; esto se ha denominado como punto, por ejemplo: el elemento fachada deteriorada se puede presentar en varias edificaciones.

En la presente investigación se trabaja en la georreferenciación, puede ser una fachada deteriorada, un cartel, un afiche, una chimenea, una tubería externa, un cableado, un poste de alumbrado deteriorado o una antena, entre otros. Los puntos se agrupan de acuerdo con sus características o similitudes en el elemento atípico correspondiente. 
La resiliencia urbana en el caso de estudio se considera por parte de los autores de esta investigación que debe estar ligada a la capacidad de las familias que residen en el sector comercial y que constituye, por ende, la comunidad de poder responder, resistir, recuperarse y adaptarse al impacto de este evento adverso (contaminación ambiental), sobre la base de sus recursos, mecanismos y estrategias comunitarias desarrollados, para la recuperación progresiva de sus medios de vida.

La gestión de riesgos es un elemento clave para la construcción de esta resiliencia y permite generar procesos comunitarios y participativos, y de empoderamiento. Así, la resiliencia comunitaria se refiere a la capacidad del sistema social, de las familias, organizaciones e instituciones, como son en este caso las asociaciones de comerciantes informales, la cámara de comercio y residentes en la zona urbana en estudio, para prevenir, hacer frente a las adversidades, reorganizarse y recuperarse tras estas afectaciones de contaminación visual de modo que mejoren sus funciones, su estructura y su identidad.

\section{CONCLUSIONES}

La zona comercial de Santo Domingo tiene problemas de contaminación visual, especialmente en la Av. 29 de mayo por poseer un alto índice de contaminación visual según los resultados obtenidos.

En la actualidad la contaminación visual se considera un factor negativo para la comunidad ya que los mismos transeúntes están conscientes de que es un problema que puede afectarles de forma directa o indirecta y que altera las condiciones normales de sus actividades cotidianas.

La propuesta presentada expone de manera clara las variables e indicadores, consideradas para el estudio específico de la imagen urbana. El tema de la imagen ha sido ampliamente estudiado por diversos autores, no obstante, es un aporte fundamental su relación con el campo de la calidad ambiental urbana, como uno de sus aspectos específicos.

También es importante señalar que la presente propuesta busca encontrar deficiencias de la calidad de la imagen tanto en sectores como en los elementos urbanos, con la intención de tratarlos desde la actuación de proyectos de diseño urbano hasta los arquitectónicos. 
En tal sentido se expone la aplicación del método en el análisis de los elementos urbanos, tomando en cuenta también las posibles intervenciones físicas que pueden y deben llevarse a cabo en los distintos sectores.

La inclusión dentro del tema de la imagen, de los valores sociales, culturales, históricos, patrimoniales; así como la búsqueda de instrumentos gráficos precisos que permitan medir la percepción que las personas tienen de un lugar, son temas que dejan el campo abierto para futuras investigaciones en el área.

\section{REFRENCIAS BIBLIOGRÁFICAS}

Arias, L., Torres, E. (2011). Gualaceo sin Contaminación Visual. Tesis de grado Licenciado en Ciencias de la Educación. Especialidad Comunicación Social Universidad de Cuenca Consultado 23 de abril 2018 http://dspace.ucuenca.edu.ec/handle/123456789/1807

Briceño, A. M. (1999). La percepción visual y la identidad formal de los objetos del espacio urbano.

Cerasi, M. M. (1977). La lectura del Ambiente. Buenos Aires: Biblioteca de Planeamiento y Vivienda, Vol. 15. Ediciones Infinito.

Diestra, N. T. (2017) La contaminación ambiental y su influencia en la salud de la población del distrito de Trujillo- la Libertad Revista CIENCIA Y TECNOLOGÍA V.13 N.3 ISSN 1810-6781 Rev. Cienc. Tecnol. 13(3): 93-102, (2017)

Etulain, J., Fisch, S., López, I. y Ponce, N. (1996). Ensayos teóricos: la ciudad y la arquitectura. Praxis y degradación urbana. El texto, el contexto y su articulación. La Plata, Argentina: Capba. Consultado el 23 de mayo 2018

GAD Santo Domingo (2015) Plan de desarrollo y ordenamiento territorial Santo Domingo de los Tsáchilas. Ecuador Consultado 3 de mayo 2018 http://gptsachila.gob.ec/documentos/LOPAIP_2016/pdyot.pdf

Matthews, T., Osorio, D., Cavallaro, A. et al. (2018). The Importance of Spatial Visual Scene Parameters in Predicting Optimal Cone Sensitivities in Routinely Trichromatic Frugivorous Old-World Primates. Front Comput Neurosci vol. 12, 15-15. 10.3389/fncom.2018.00015 Consultado el 3 de mayo 2018 http://qmro.qmul.ac.uk/xmlui/handle/123456789/36665 
Rivera C., Gabriel H. (2013). Elementos atípicos y contaminación visual urbana en un sector de la zona centro de Bogotá. Épsilon (21), 83-105.

Trujillo, F. (2010). Diagnóstico de la contaminación visual urbana en la localidad de La Candelaria de Bogotá D.C. (tesis de pregrado inédita). Universidad de La Salle, Bogotá, Colombia.

UNISDR (2017) Cómo desarrollar ciudades más resilientes. Manual para líderes de los gobiernos locales Ginebra, Versión de 2017 Consultado 12 de abril 2018 http://www.eird.org/americas/

Universidad Nacional de Colombia. (2008). Estatuto del espacio público Municipio de Palmira. Convenio interinstitucional № 040, Municipio de Palmira, Secretaría de Planeación, y Universidad Nacional de Colombia sede Palmira. Unimedios. Publicación de la Unidad de Medios de Comunicación-Unimedios de la Universidad Nacional de Colombia, 28 de enero. Recuperado de http://historico.agenciadenoticias.unal.edu.co/articulos/universidad/universidad_ 20080128_palmira.html. 